\title{
Oxytetracycline recovery from aqueous media using computationally designed molecularly imprinted polymers
}

\author{
Rosalía Rodríguez-Dorado ${ }^{1}$, Antonia M. Carro ${ }^{2}$, Iva Chianella ${ }^{3}$, Kal Karim ${ }^{4}$, Angel \\ Concheiro $^{1}$, Rosa A. Lorenzo ${ }^{2}$, Sergey Piletsky ${ }^{4}$, Carmen Alvarez-Lorenzo ${ }^{1 * *}$
}

${ }^{1}$ Departamento de Farmacia y Tecnología Farmacéutica, R+DPharma Group (GI-1645),

Facultad de Farmacia, and Health Research Institute of Santiago de Compostela (IDIS),

Universidade de Santiago de Compostela, 15782-Santiago de Compostela, Spain.

2 Departamento de Química Analítica, Nutrición y Bromatología, Facultad de Química, and Health Research Institute of Santiago de Compostela (IDIS), Universidade de Santiago de Compostela, 15782-Santiago de Compostela, Spain.

${ }^{3}$ Cranfield University, Bedford MK45 4DT, England, United Kingdom.

${ }^{4}$ Chemistry Department, College of Science and Engineering, University of Leicester, LE1 7RH

*Corresponding author. E-mail address: carmen.alvarez.lorenzo@usc.es; Tel: 34 981563100 ext. 15239, Fax: 34981547148. 


\begin{abstract}
Polymers for recovery/removal of antimicrobial agent oxytetracycline (OTC) from aqueous medium were developed using computational design and molecular imprinting. 2-Hydroxyethyl methacrylate (HEMA), 2-acrylamide-2-methylpropane sulfonic acid (AMPS) and mixtures of both were chosen according to their predicted affinity for OTC and evaluated as functional monomers in imprinted (MIPs) and non-imprinted (NIPs) polymers. Two levels of AMPS were tested. After bulk polymerization, the polymers were crushed as particles $(200-1000 \mu \mathrm{m})$. A pressurized liquid extraction (PLE) procedure was implemented for template removal using a low amount of methanol (less than $20 \mathrm{~mL}$ in each extraction), and few extractions (12-18 for each polymer) in a short period of time (20 minutes per extraction). Particles size distribution, microporous structure and capacity to rebind OTC from aqueous media were evaluated. Adsorption isotherms obtained from OTC solutions (30 to $110 \mathrm{mg} \mathrm{L}^{-1}$ ) revealed that the polymers prepared with AMPS have the highest affinity for OTC. The uptake capacity depended on the ionic strength as follows: purified water $>$ saline solution $(0.9 \% \mathrm{NaCl})>$ seawater $(3.5 \% \mathrm{NaCl})$. Polymer particles containing AMPS as functional monomer showed a remarkable ability to clean water contaminated with OTC. Usefulness of developed stationary phase for solid phase extraction (MISPE) was also demonstrated.
\end{abstract}

Keywords: computational design; molecularly imprinted polymer; oxytetracycline; rebinding isotherm; solid-phase extraction; groundwater remediation. 


\section{Introduction}

The widespread production and use of antibiotics for human and veterinary medicine has led to their frequent presence as pollutants of soils and surface water [1-4]. Tetracyclines, a broad-spectrum antibiotic family effective against Gram-positive and Gram-negative bacteria and some protozoa, are extensively applied for prophylaxis and treatment of infections in livestock industry. After animals fed, non-absorbed dose directly comes into the environment, whereas the fraction absorbed is metabolized and eliminated through urine [5,6]. Degradation of tetracyclines in soil and water is very slow, and only strong oxidizing agents are able to efficiently degrade them [2,7-8]. Presence of tetracyclines in the environment causes sensitization problems and favors the increasing resistance shown by pathogenic microorganisms [3,9]. Consequently, methods suitable for the removal of tetracyclines from the environment are in high demand [1].

Molecularly imprinted polymers (MIPs) already occupy an important position among solid phases employed for selective capture/retention of analytes [10,11]. Molecular imprinting technology aims to create tailored binding sites by synthesizing polymer network in the presence of a target substance which acts as a template. Functional monomers with the best capacity for interaction with the functional groups of the template are selected for polymer preparation. Polymerization and cross-linking make the most favorable arrangement of the monomers around template molecule permanent. Once the polymer network is formed, the template molecule must be removed to generate free binding sites. The binding site conformation and thus the capacity of the polymer to selectively recognize the target molecules depends on the monomers nature, the functional monomer:template stoichiometry, and the conditions in which the polymerization is carried out [10]. A number of MIPs have been already developed for 
the recovery of tetracyclines from different media, mainly for analytical purposes such as sample concentration (molecularly imprinted solid-phase extraction, MISPE) [1235]. The rational criteria were not applied for the selection of MIP composition in these instances and most of traditional MIPSE polymers still rely on the use of acrylic acid or methacrylic acid as functional monomers [10]. Molecular modelling approaches allow identification of suitable functional monomers and adequate template:monomer stoichiometry and offer remarkable advantages [36,37]. Analysis of the interactions between template molecules and functional monomers, under the polymerization conditions, provides an information about efficient functional monomers that can form complexes with a given template (molecular modelling) and about the binding energies and stoichiometry of the complexes as well as the type of interaction (hydrogen bonding, self-association, $\pi-\pi$ stacking, ionic interactions). This approach has been used successfully for developing highly efficient MIPs for analytical applications [38]. Removal of the template after polymerization, without altering the inner structure of the MIP, is also a very important issue [39]. Although still barely used for this purpose, techniques that use pressurized solvents may provide higher yields in shorter time and reduce use of solvents as compared with conventional techniques such as Soxhlet extraction. Pressurized Liquid Extraction (PLE) performs the extractions under elevated temperatures $\left(50-200^{\circ} \mathrm{C}\right)$ and pressures $(10-14 \mathrm{MPa})$ decreasing the viscosity and the surface tension of the solvent [40], which in turn favors solvent penetration into the polymer networks. Relatively high temperatures (above the boiling temperature of the solvent) also facilitate analyte solubilization and attenuate analyte-network interactions. The use of high pressure facilitates the extraction of analytes trapped in pores. A complete extraction with PLE may be obtained with a low volume of solvent and in a 
short period of time (10-25 min). These advantages can make this technique suitable for template removal from MIPs [39-42].

In the present work, polymers with high affinity for oxytetracycline (OTC) were designed using molecular modelling. The optimal functional monomers were selected from a virtual library of twenty five monomers (Figure 1) often used in preparation of MIPs. MIPs and the corresponding non-imprinted polymers (NIPs) were prepared with various ratios of chosen functional monomers. As other novel issue, a simple PLE procedure was implemented for template removal. Then, microporous structure and OTC uptake capacity were characterized in detail. Adsorption isotherms at equilibrium in aqueous media of different salt concentration and performance as MISPE were evaluated. Conditions suitable for elution and recovery of the retained OTC were also investigated.

\section{Materials and methods}

\subsection{Materials}

Oxytetracycline $\mathrm{HCl}$ ( $\geq 95 \%$ ), 2-acrylamido-2-methylpropane sulfonic acid (AMPS) and ethylene glycol dimethacrylate (EGDMA) were from Sigma-Aldrich (St. Louis, Mo, USA); 1,1'-azobis(isobutyronitrile) (AIBN) from Acros (Geel, Belgium); 2hydroxyethyl methacrylate (HEMA) and hydrochloric acid from Merck (Darmstadt, Germany); dimethylsulfoxide (DMSO) from Scharlau Chemicals (Barcelona, Spain); methanol HPLC gradient from Prolabo (Barcelona, Spain); and sodium chloride ( $\mathrm{NaCl}$ ) from Panreac (Barcelona, Spain). Purified water was obtained by reverse osmosis (MilliQ ${ }^{\circledR}$, Millipore). 


\subsection{Synthesis of MIPs}

The rational design of MIPs was carried out on a PC running Linux operating system executing the software package SYBYL 7.3 (Tripos Inc., St. Louis, MO, USA). The design protocol involved three steps: 1) design of functional monomer database; 2) design of molecular model of template to be screened; and 3) screening using a LEAPFROG $^{\mathrm{TM}}$ algorithm. The library designed for this project contained 25 commonly used functional monomers (Figure 1), which possess polymerizable residues and residues capable of interacting with a template through electrostatic and van der Waals forces. Preferred monomers are those that are able to interact with the template through non-covalent interactions and that are commercially available and could be polymerized by heating or photo-initiation. The charges for each atom of each monomer were calculated, and the structures of the monomers refined using molecular mechanics methods. For all the monomers in the database energy was minimized individually to a value of $0.001 \mathrm{kcal} \mathrm{mol}^{-1}$ [43]. For the second step, the structure of OTC was modeled in a similar manner to that of the monomers. The charges for each atom on the template were calculated and the structures refined using molecular mechanics. Energy minimization was performed to a value of $0.001 \mathrm{kcal} \mathrm{mol}^{-1}$ (energy minimized using the dielectric constant value of DMSO to get stable conformations), and the model was then used for the design of MIPs. The LEAPFROG ${ }^{\mathrm{TM}}$ algorithm (60,000 iterations) was applied to screen the library of functional monomers for their possible interactions with the template. The estimated interaction energies between OTC and the best candidate monomers included in the virtual library are shown in Table 1.

Once the most adequate monomers were identified, two series of polymers were prepared with the compositions shown in Table 2. Appropriate amounts of the monomers were dissolved in DMSO under magnetic stirring. In separate, OTC amounts 
were weighed in vials and then aliquots of the monomers solution were added. Other aliquots without OTC were used to prepare the corresponding NIPs. The resultant solutions were placed in an ultrasound bath during $1 \mathrm{~min}$ in order to remove some oxygen $[19,23]$, without damage of OTC. Polymerization was carried out during 48 hours at $50^{\circ} \mathrm{C}$ in an oven. The obtained polymers were crushed in a porcelain mortar and sieved to separate the $200-1000 \mu \mathrm{m}$ particle size fraction.

\subsection{Template removal}

Polymer particles (0.8-1.9 g) were subjected to extraction with methanol at high pressure and temperature in a pressurized liquid extraction (PLE) apparatus (ASE 150, Dionex, Thermo Scientific, Spain) under the following conditions: pre-heat $5 \mathrm{~min}$; temperature $80^{\circ} \mathrm{C}$; pressure 1500 psi (103.42 bar); extraction time $5 \mathrm{~min}$; flush volume $80 \%$; number of static cycles 2; and solvent methanol. Each sample underwent between 12-18 extractions and each extract was separated and kept at $4^{\circ} \mathrm{C}$ protected from light. OTC concentration was quantified from absorbance measurements at $354 \mathrm{~nm}$ (Agilent 8453 spectrophotometer, Germany). Then, each polymer sample was dispersed in 10 $\mathrm{mL}$ of $\mathrm{MeOH}: \mathrm{HCl} 1 \mathrm{~N} \mathrm{50:50(v/v)} \mathrm{in} \mathrm{Falcon} \mathrm{tubes,} \mathrm{under} \mathrm{magnetic} \mathrm{stirring,} \mathrm{for} 1$ hour. Centrifugation was applied and the supernatant was spectrophotometrically quantified at $354 \mathrm{~nm}$. After that, polymers were transferred to $10 \mathrm{~mL}$ of $\mathrm{MeOH}$ in Falcon tubes and similarly processed. Finally, polymers were subjected to two extractions more using PLE as described above. All the extracts were filtered through $0.2 \mu \mathrm{m}$ Nylon membranes, and the absorbance measured at $354 \mathrm{~nm}$. Finally, polymers were dried for

72 hours at $70^{\circ} \mathrm{C}$, and 12 hours at room temperature in a vacuum desiccator. OTC removed from MIPs was quantified using a calibration curve (obtained with standard aqueous solutions) and subtracting the absorbance recorded for the extracts of the nonimprinted polymers (NIPs) caused by un-reacted monomers. The amount of OTC 
remaining in the polymers after the removal step was quantified as the difference between total OTC added for synthesis and total OTC removed.

\subsection{Polymers physical characterization}

Optical microscopy. Particle size distribution was characterized using an Olympus SZCTV magnifying glass (Japan) connected to a DP12 digital microscope camera. Samples of each polymer were placed on Petri dishes under the magnifying glass. The zoom lens was 1.5X. Images of 600 particles were treated using AnalySIS software (Soft Imaging System GmbH, Münster, Germany). Feret maximum, Feret minimum and Feret mean diameter data from each particle were measured, and particle size $(\mu \mathrm{m})$ distribution was analyzed.

Nitrogen adsorption porosimetry. Specific surface area, pore volume and average pore diameter data of each polymer were obtained from $\mathrm{N}_{2}$ adsorption isotherms at $77 \mathrm{~K}$, recorded in an ASAP 2000 apparatus (Micromeritics, Norcross, GA, USA) applying BET theory and mathematical models as previously described [44].

\subsection{OTC rebinding isotherms}

Rebinding of OTC was tested in three different aqueous media: purified water, $0.9 \%$ $\mathrm{NaCl}$ and $3.5 \% \mathrm{NaCl}$ solutions. Polymer samples $(10 \mathrm{mg})$ were dispersed in $10 \mathrm{~mL}$ of drug solutions $\left(30,50,70,90\right.$ and $\left.110 \mathrm{mg} \mathrm{L}^{-1}\right)$ in Falcon tubes of $15 \mathrm{~mL}$ under magnetic stirring. After 24 hours, tubes were centrifuged at $5000 \mathrm{rpm}$ at $20^{\circ} \mathrm{C}$ for $10 \mathrm{~min}$. The absorbance of the supernatant was measured at $354 \mathrm{~nm}$ in order to calculate the remaining free OTC $\left(\mathrm{mg} \mathrm{mL}^{-1}\right)$. OTC adsorbed $\left(\mathrm{mg} \mathrm{g}^{-1}\right)$ was estimated as the difference between initial and final OTC concentration in the medium and referred to each polymer weight. The Freundlich model was fitted to the adsorption isotherms [45]. 


\subsection{Molecularly imprinted solid-phase extraction (MISPE)}

Polymers prepared with the highest contents of functional monomers (series b in Table

2) were evaluated on their ability to retain and elute OTC under dynamic conditions. $100 \mathrm{mg}$ of each dried polymer were packed into $3 \mathrm{~mL}$ SPE cartridges (Supelco, USA) with one frit at each end, and the conditioning step was carried out with $2 \mathrm{~mL}$ of methanol and $2 \mathrm{~mL}$ of purified water. An OTC solution $\left(0.0171 \mathrm{mg} \mathrm{mL}^{-1} ; 40 \mathrm{~mL}\right)$ was passed through the cartridge in the sequence of 20 loads of $2 \mathrm{~mL}$. Then, the elution step was carried out with methanol (4 x $2 \mathrm{~mL})$ and $\mathrm{MeOH}: \mathrm{HCl}(0.1 \mathrm{M})$ 50:50 v:v (6 x 2 $\mathrm{mL})$. Percolations of both steps were collected in Eppendorf tubes and the absorbance measured at $354 \mathrm{~nm}$ (Agilent 8453 spectrophotometer, Germany).

\section{Results and discussion}

\subsection{Synthesis of MIPs}

Tetracyclines are amphoteric compounds with three $\mathrm{pKa}$ values $(3.57,7.49,9.44)$. Thus, OTC behaves as zwitterion at $\mathrm{pH}$ between $\mathrm{pKa}_{1}$ and $\mathrm{pKa}_{2}$ values [2]. We observed that this drug strongly inhibits UV-initiated polymerization. Moreover, it degrades at high temperature [46], which limits the polymerization approach to quite mild conditions. On the other hand, for an efficient removal from contaminated aqueous medium, functional monomers that can strongly capture OTC from polar environments are required. The rational design protocol involved screening of functional monomer database (Figure 1) using a LEAPFROG ${ }^{\mathrm{TM}}$ algorithm against molecular model of template (energy minimized using the dielectric constant value of DMSO to get stable conformations). This step gives a very good indication of the best monomer(s) for polymer preparation in a given solvent. Monomers giving the highest binding energies represent the best 
candidates for polymer preparation and for forming the strongest complexes with the template (Table 1). In our work the strongest binding energy was observed for a common cross-linking agent, N,N-methylenebis(acrylamide). This indicates that the polymer networks cross-linked with this monomer would exhibit high affinity disregarding whether they were imprinted or not. AMPS, which ranked second in affinity, has the additional advantage that its sulfonic group remains ionized in a wide range of $\mathrm{pH}$, even under acidic conditions ( $\mathrm{pKa} \sim 1.0)$. Figure 2 shows the interaction between OTC and AMPS monomer. Ethyleneglycol methacrylate phosphate and N-(2(phenylamino)ethyl methacrylamide are not commercially available and for this reason they were excluded from further work. Regarding other commercially available monomers that may lead to environmentally friendly networks, HEMA appeared as a suitable candidate. HEMA may make the networks hydrophilic and thus facilitate water diffusion, which is required for the access of OTC molecules from the contaminate environment to the binding points in the network. Moreover, although with less binding energy than AMPS, HEMA can also interact with OTC. Interestingly, neither acrylic acid nor methacrylic acid, commonly used in preparation of OTC-imprinted networks [14-35], were ranked in the first ten positions.

Thus, NIPs and MIPs were prepared combining AMPS and HEMA, and using EGDMA as a cross-linker that showed very little non-specific affinity for OTC. EGDMA seems to be ideal cross-linker that allows elucidating the effect of the functional monomer and molecular ratio on the rebinding of OTC and the imprinting effect. The drug and the monomers (at two levels of template:functional monomer 1:4.5 and 1:9 molar ratio; Table 2) were dissolved in DMSO and the polymerization was carried out at $50^{\circ} \mathrm{C}$ for 48 hours in order to minimize template degradation. Then, the polymer blocks were crushed and sieved to produce $200-1000 \mu \mathrm{m}$ particles. 


\subsection{Template removal}

Solvents employed in PLE technique can be the same as those normally used for the standard liquid extraction techniques like Soxhlet or sonication [40]. In the present work methanol was chosen because of its polar character and the high solubility of OTC in this solvent. Temperature and pressure are critical variables in PLE performance. A pressure of 1500 psi (the pressure usually used in PLE is 1000-2000 psi; i.e., 69-138 bar) allowed working at $80^{\circ} \mathrm{C}$ (above the methanol boiling point under normal conditions) while still having the solvent as liquid [40]. Both MIPs and NIPs were processed similarly in order to discard differences in OTC binding due to different processing conditions [39]. Each polymer sample underwent 12-18 extractions (20 min/extraction). Cumulative values of OTC removed per gram of dry polymer are shown in Figure 3. Release of non-reacted monomers (as observed for NIPs) minimally interfered in OTC quantification at the chosen wavelength (Table 3).

OTC was removed in greater amount and more rapidly from MIP series 2 (based on HEMA) compared to MIP series 4 (based on AMPS) and 6 (based on AMPS and HEMA). For MIP series 2, OTC removal using methanol as solvent in the PLE was almost complete with only two extractions, which means that the OTC-polymer hydrophobic interaction and hydrogen bonding are easily broken using methanol. With increase in the content of AMPS (which can establish ionic interactions with OTC), the removal became slower and more extractions were required. Assuming that $18.2 \mathrm{mg}$ of OTC removed per gram of 2a-MIP is the total amount of drug incorporated in the polymer, PLE treatment of 4a-MIP and 6a-MIP only allowed for the removal of $46.7 \%$ and $41.2 \%$ of the drug, respectively. Meanwhile, in the case of $2 \mathrm{~b}-\mathrm{MIP}$ only $17.2 \mathrm{mg} \mathrm{g}^{-1}$ of OTC were removed, whereas the total amount of drug removed through PLE from 4b-MIP and 6b-MIP was $28.7 \%$ and $33.0 \%$, respectively. These differences are related 
to the high affinity of the functional monomer for the drug; therefore, in polymer "series a" containing smaller amount of AMPS, removal of the template was easier than in polymers of "series b".

To improve the extraction yield, all polymers were then submitted to an additional step of template removal in Falcon tubes with a methanol:HCl mixture followed by pure methanol, and then two more extractions with methanol in the PLE equipment. This combination of solvents (particularly $1 \mathrm{~N} \mathrm{HCl}$ ) was expected to weaken the ionic interactions between the drug and the polymers prepared with AMPS monomer. These additional extractions were carried out in Falcon tubes because $\mathrm{HCl}$ cannot be used in the ASE 150 instrument. The additional amounts of OTC removed with this procedure are shown in Table 3. Overall, nearly $60 \%$ and $50 \%$ template was removed from MIP "series a" and "b", respectively. This means that a relevant proportion of OTC still remains in the MIPs containing AMPS even after this extraction process. Alternatively, OTC can be partially destroyed during polymerization which might affect its detection by spectrophotometry. Permanent retention of the template is a quite relevant issue that is commonly undervalued or omitted in most reports on imprinted polymers [47]. As stated in most papers, the OTC-MIPs underwent extraction until no more OTC was detected in the eluent, but we observed that this does not necessarily mean that OTC was completely removed. In fact, nearly $40 \%$ of the OTC in 4-MIP and 6-MIP are either destroyed or remained permanently in the polymer. In essence the true reason for OTC disappearance in extract is not important as long as leaching of the template from the polymer in binding experiments cannot influence analytical data.

\subsection{Polymers physical characterization}


Synthesized polymers had particle size between 200 and $1000 \mu \mathrm{m}$ (size distributions in Electronic Supplementary Material Figures S1 and S2). Nitrogen adsorption porosimetry revealed that the polymers had pores in the mesoporous range (diameter between 2-50 nm) with an average pore diameter between 6 and $9 \mathrm{~nm}$ (Table 3). The molecular size of OTC (estimated with Chem3D software, CambridgeSoft, PerkinElmer, Waltham, MA, USA) is $1.32 \times 0.70 \mathrm{~nm}$; therefore the dimensions of the pores may allow easy penetration of OTC molecules in and out of the polymer network. Typical values for surface area of the imprinted polymers are in the range of 100 to 500 $\mathrm{m}^{2} \mathrm{~g}^{-1}[30,48]$. In general, polymers of "series a" had specific surface area and pore volume larger than those of "series b". Compared to NIPs, MIPs containing more AMPS had lower surface area and pore volume which can be related to closed pores in the imprinted polymers.

\subsection{OTC rebinding isotherms}

OTC adsorption studies were carried out in aqueous solutions with different ionic strength trying to mimic the saline environments where this drug can be found: water (purified water), $0.9 \% \mathrm{NaCl}$ solution (mimicking the ionic strength of biological fluids) and $3.5 \% \mathrm{NaCl}$ solution (resembling the salt concentration of seawater). OTC captured by each polymer in the three different media is shown in Figure 4.

Polymers containing only HEMA as functional monomer (series 1 and 2) showed low OTC adsorption ability. Interestingly, the binding increased with the increment of $\mathrm{NaCl}$ concentration in the aqueous media. This finding indicates that OTC interacts with the polymer mainly through hydrophobic forces that are favored by the salting-out effect 
and the screening of the drug molecules charges, avoiding repulsion phenomena. As a consequence, NIPs and MIPs performed similarly, which supports this hypothesis.

Polymers containing AMPS solely (series 3 and 4) or combined with HEMA (series 5 and 6) trapped notably higher amounts of OTC, but mainly in media without salt. It is important to highlight the fact that AMPS-bearing polymers $(10 \mathrm{mg})$ dispersed in the OTC solution of the lowest concentration tested $\left(30 \mathrm{mg} \mathrm{L}^{-1} ; 10 \mathrm{~mL}\right)$ were able to uptake the whole amount of drug. This concentration is similar to that found at the discharge point of waste from antibiotics production plants and well above the value recorded in inland waters contaminated with the drug $\left(0.6 \mathrm{mg} \mathrm{L}^{-1}\right)$ [9]. In non-saline aqueous media (Figure 4), polymers did not reach saturation even when they were exposed to higher levels of OTC, capturing approx. $80 \mathrm{mg}\left(\approx 170\right.$ micromol $\left.\mathrm{g}^{-1}\right)$ of drug per gram. The whole content in AMPS was 226 micromol g $\mathrm{g}^{-1}$ and 452 micromol $\mathrm{g}^{-1}$ in the case of polymers "series a" and "b", respectively, which suggests that the binding points are not saturated. Polymers containing AMPS demonstrated notably diminished OTC adsorption when salt concentration increased (Figure 4). This is directly related to the ionic interaction mechanism of the drug with the functional monomer AMPS, which is affected by the presence of salts. Generally no significant differences were observed between MIPs and NIPs, except that 3-NIP loaded more OTC than 4-MIP probably due to the difficulty in removing template molecules from the polymer.

Previous papers on OTC-MIPs prepared with acrylic acid or methacrylic acid reported binding isotherms with saturation values in the 2-30 micromol $\mathrm{g}^{-1}$ range (i.e., $1-15 \mathrm{mg} \mathrm{g}^{-}$ 1) $[14,21,25,30,31]$, which are similar to or even lower than those obtained for the developed HEMA-based polymers (1a/b-NIP and 1a/b-MIP) (Figure 4). Core-shell magnetic-methacrylic acid particles have been shown to take up $24 \mathrm{mg}$ tetracycline per gram from aqueous medium [49]. Higher rebinding was observed for MIPs prepared in 
methanol with template:methacrylic acid 1:10 molar ratio (which seems to be quite large ratio and may lead to non-imprinted cavities) that captured up to 113 micromol $\mathrm{g}^{-1}$ $\left(\approx 50 \mathrm{mg} \mathrm{g}^{-1}\right)$ [24], and for a molecularly imprinted organic-inorganic hybrid composite material that captured $60 \mathrm{mg}$ OTC per gram from dichloromethane/methanol (92:8 v:v) as rebinding solvent [23]. So far, activated charcoal (of extremely high surface area) ranks first in ability to adsorb OTC (more than $400 \mathrm{mg} \mathrm{g}^{-1}$ ) [50], but the process is almost irreversible and therefore this material is not useful for analytical purposes. Thus, polymers prepared with AMPS either solely or combined with HEMA (namely, the monomers identified by molecular modelling as the most suitable ones for OTC binding) appear to be advantageous compared to systems investigated so far. Detrimental effects of the ionic strength of the aqueous medium on OTC adsorption have been also recorded for river sediments and soil components, which retained 0.5$0.8 \times 10^{-3} \mathrm{mg} \mathrm{g}^{-1}$ in the presence of $0.1 \% \mathrm{NaCl}$ [51] or $2 \mathrm{mg} \mathrm{g}^{-1}$ in $0.05 \% \mathrm{NaCl}$ aqueous solution [2]. Recently, MIPs for tetracycline prepared with template:methacrylic acid 1:7 molar ratio have shown a decrease in the rebinding uptake from 1.1 micromol g $\mathrm{g}^{-1}$ in water to 0.9 micromol $\mathrm{g}^{-1}$ in $0.1 \mathrm{M} \mathrm{NaCl}(0.55 \%)$, which confirmed the prevalence of ionic interactions especially at high drug concentration [33].

To gain further insight into the effect of the monomers nature and the presence of template during polymerization, adsorption isotherms were analyzed according to the Freundlich model [45]:

$$
\log B=m \cdot \log F+\log a
$$

where $\mathrm{B}\left(\mu \mathrm{mol} \mathrm{g}^{-1}\right)$ and $\mathrm{F}(\mu \mathrm{M})$ refer to the bound and free OTC concentration, respectively, and $m$ and $a$ are adjustment parameters. The $a$ parameter provides a measure of the number of binding points and the average affinity of the polymer. The $m$ 
parameter is an index of binding points heterogeneity: values close to 1 means that all binding sites are similar from an energetic point of view, while values close to 0 indicates heterogeneous binding points. Results from the fit to the Freundlich model (Table 4) indicated that in water the higher $m$ values correspond to NIPs and MIPs prepared with HEMA, suggesting that this monomer provides homogeneous binding sites disregarding the effect of molecular imprinting. Polymers prepared with AMPS either solely or combined with HEMA led to notably lower $m$ and higher $a$ values which confirms the creation of high affinity binding points. As mentioned above, OTC may interact with the polymers through hydrophobic, hydrogen bonding and electrostatic interactions. Since only one strong electrostatic interaction is expected to occur in the binding of each OTC molecule, differences between MIPs and NIPs are minor. This finding is in good agreement with previous reports that indicate that the imprinting effect is more remarkable when the functional monomers interact with the template by means of weak multiple-point interactions [43]. Also for polymers prepared with AMPS and AMPS+HEMA, affinity for OTC diminished 10-fold or 20-fold when the medium changed from water to $0.9 \%$ or $3.5 \% \mathrm{NaCl}$ saline aqueous media, which once again confirms the prevalence of the electrostatic interactions in the binding. The opposite effect was observed for polymers containing HEMA, which seems to favor hydrophobic interactions with the template.

\subsection{Molecularly imprinted solid-phase extraction (MISPE)}

Since adsorption requires at least 24 hours till equilibrium reached, the next step was to evaluate the suitability of the synthesized polymers for cleaning of contaminated water under dynamic conditions and for sample concentration under MISPE mode [10]. To do 
that, an OTC solution $\left(0.0171 \mathrm{mg} \mathrm{mL}^{-1} ; 40 \mathrm{~mL}\right)$ was passed through $3 \mathrm{~mL} \mathrm{SPE}$ cartridges containing $100 \mathrm{mg}$ polymer, in the sequence of 20 loads of $2 \mathrm{~mL}$. Polymers with the highest content in functional monomer were tested ("series b" of Table 2).

Polymers prepared only with HEMA, 1b-NIP and 2b-MIP, retained smaller amounts of OTC (Figure 5) and, as a consequence, OTC concentration in the percolated water remained mostly unaltered (Figure 6). In polymers containing only AMPS, the nonimprinted polymer $3 b-N I P$ was the one that retained the highest amount of drug due to the strong electrostatic interactions with all available binding points, and allowed for the retention of nearly $90 \%$ OTC in the aqueous medium that flowed through the SPE cartridges. Regarding polymers containing both monomers, the 6b-MIP retained high amounts of OTC due to the presence of imprinted cavities with the appropriate size to bind this drug and with suitable chemical groups to establish with it ionic and hydrophobic interactions and hydrogen bonds (Figure 5). Interestingly, polymers bearing HEMA as the only functional monomer showed a progressive decrease in the binding of OTC after subsequent loads, whereas polymers prepared with AMPS or AMPS+HEMA showed an almost constant binding capacity (within experimental errors) along the 20 loads, in good agreement with the high saturation levels exhibited by these polymers under static conditions (Figure 4). The different performance of polymers prepared with different functional monomers is reflected in the amount of OTC accumulated in the cartridges, which increased almost linearly for $3 b-N I P, 4 b-$ MIP, 5b-NIP and 6b-MIP (Figure 6).

Recovery of OTC from the polymers was carried out using first methanol and then methanol:HCl 0.1M 50:50 v:v. In the case of 1b-NIP and 2b-MIP, 80\% OTC was eluted using only three aliquots $(2 \mathrm{~mL})$ of methanol (Figure $7 \mathrm{a})$, which is in good agreement with the hydrophobic (or hydrogen bonding) interactions that OTC can establish with 
HEMA-based polymers. In contrast, methanol solely was not able to desorb OTC from polymers prepared with AMPS or AMPS+HEMA, and a mixture of $\mathrm{MeOH}: \mathrm{HCl} 0.1 \mathrm{M}$ was required (Figure $7 \mathrm{~b}$ and $7 \mathrm{c}$ ). It has been previously observed that tetracyclines are better recovered from MISPE phases (prepared using methacrylic acid as functional monomer) using elution solvents in a $\mathrm{pH}$ range of 2-3 [21,25], probably because the protonation of OTC makes the drug more hydrophilic and the ions in the medium attenuate the ionic interactions. Interestingly, in our study more rapid and complete recovery of OTC was observed for 4-MIP and 6-MIP compared to the non-imprinted counterparts, proving their utility as stationary phase in SPE. These differences can be explained by the complex nature of the interaction of OTC with imprinted sites which can be disrupted in appropriate solvent.

\section{Conclusion}

Selection of functional monomers by molecular modelling allowed for notable improvement in the performance of polymer networks aimed to remove pollutants from contaminated environment. HEMA, AMPS and its mixture endowed polymer particles with remarkably high affinity for OTC. Using the drug as a template during polymerization did not significantly modify the total binding ability of the polymers (MIPs and NIPs behaved similarly), which can be attributed to (i) the high affinity of selected monomers to the template that cannot be further improved by molecular imprinting, and (ii) the retention of an important amount of template in the MIPs even after several cycles of PLE. Both AMPS-based MIPs and NIPs were able to completely remove OTC from water contaminated with OTC levels similar to those of industrial effluents. Interestingly, HEMA-based polymers are suitable for OTC recovery from 
saline medium of high ionic strength, as hydrophobic interactions are fortified by the salting out effect. Regarding performance under dynamic conditions (in the SPE cartridges), MIPs and NIPs showed similar ability to retain the drug, but elution from MIPs was easier, enabling $100 \%$ recovery after few elution cycles. Overall, molecular modelling enables the identification of strong functional monomers and thus the preparation of polymers with high binding capacity even in the absence of template polymerization. NIPs have the advantages of faster cleaning and avoidance of risk of template bleeding during use. However, MIPs still appear to be advantageous regarding rapidness of loading/elution because the creation of specific cavities for hosting the drug that can be affected by change of the solvent during elution of the template molecules.

Funding: Work supported by MINECO (SAF2014-52632-R and AGL2014-53647-R) and Ministry of Interior-DGT (SPIP2015-01838) Spain and FEDER.

Conflicts of interest: The authors declare that they have no conflict of interest

Electronic Supplementary Material. Particle size distribution of 1a-NIP, 2a-MIP, 3aNIP, 4a-MIP, 5a-NIP and 6a-MIP polymers, and 1b-NIP, 2b-MIP, 3b-NIP, 4b-MIP, 5bNIP and 6b-MIP polymers

\section{References}

[1] Moreno-Bondi MC. Antibiotics in food and environmental samples. Anal Bioanal Chem. 2009;395:875-876.

[2] Peng FJ, Zhou LZ, Ying GG, Liu YS, Zhao JL. Antibacterial activity of the soilbound antimicrobials oxytetracycline and ofloxacin. Environ Toxicol Chem. 2014;33:776-783. 
[3] Martínez JL. Environmental pollution by antibiotics and by antibiotic resistance determinants. Environ Pollut. 2009;157:2893-2902.

[4] Gadipelly C, Perez-Gonzalez A, Yadav GD, Ortiz I, Ibañez R, Rathod VK, Marathe KV. Pharmaceutical industry wastewater: review of the technologies for water treatment and reuse. Ind Eng Chem Res. 2014;53:11571-11592.

[5] Zuccato E, Calamari D, Natangelo M, Fanelli R. Presence of therapeutic drugs in the environment. Lancet 2000;355:1789-1790.

[6] Rigos G, Smith P. A critical approach on pharmacokinetics, pharmacodynamics, dose optimisation and withdrawal times of oxytetracycline in aquaculture. Rev Aquacult. 2015;7:77-106.

[7] Li ZJ, Fan FF, Long J. Effects of soil temperature on degradation of oxytetracycline in soils. Res J Chem Environ. 2013;17:56-61.

[8] Pouliquen H, Delépée R, Larhantec-Verdier M, Morvan ML, Le Bris H. Comparative hydrolysis and photolysis of four antibacterial agents (oxytetracycline oxolinic acid, flumequine and florfenicol) in deionised water, freshwater and seawater under abiotic conditions. Aquacult. 2007;26223-28.

[9] Li D, Yu T, Zhang Y, Yang M, Li Z, Liu M, Qi R. Antibiotic resistance characteristics of environmental bacteria from an oxytetracycline production wastewater treatment plant and the receiving river. Appl Environ Microbiol. 2010;76:3444-3451.

[10] Tamayo FG, Turiel E, Martín-Esteban A. Molecularly imprinted polymers for solid-phase extraction and solid-phase microextraction: Recent developments and future trends. J Chromatogr A 2007;1152:32-40.

[11] Lorenzo RA, Carro AM, Concheiro A, Alvarez-Lorenzo C. Stimuli-responsive materials in analytical separations. Anal Bioanal Chem. 2015;407:4927-4948. 
[12] Caro E, Marce RM, Cormack PAG, Sherrington DC, Borrull F. Synthesis and application of an oxytetracycline imprinted polymer for the solid-phase extraction of tetracyclines antibiotics. Anal Chim Acta. 2005;552:81-86.

[13] Turiel E, Martín-Esteban A. Molecularly imprinted polymers for sample preparation: a review. Anal Chim Acta. 2010;668:87-99.

[14] Kong JH, Wang YZ, Nie C, Ran D, Jia XP. Preparation of magnetic mixed templates molecularly imprinted polymer for the separation of tetracycline antibiotics from egg and honey samples. Anal. Methods. 2012;4:1005-1011.

[15] Xiong Y, Zhou HJ, Zhang ZJ, He DY, He C. Molecularly imprinted on-line solidphase extraction combined with flow-injection chemiluminiscence for the determination of tetracycline. Analyst. 2006;131:829-834.

[16] Caro E, Marce RM, Cormack PAG, Sherrington DC, Borrull F. Synthesis and application of an oxytetracycline imprinted polymer for the solid-phase extraction of tetracyclines antibiotics. Anal Chim Acta. 2005;552:81-86.

[17] Sun XL, He XW, Zhang YK, Chen LX. Determination of tetracyclines in food samples by molecularly imprinted monolithic column coupling with high performance liquid chromatography. Talanta 2009;79:926-934.

[18] Jing T, Gao XD, Wang P, Wang Y, Lin YF, Hu XZ, Hao QL. Determination of trace tetracycline antibiotics in foodstuffs by liquid chromatography-tandem mass spectrometry coupled with selective molecular-imprinted solid-phase extraction. Anal Bioanal Chem. 2009;393:2009-2018.

[19] Wang HT, Zhao HM, Quan X, Chen S. Electrochemical determination of tetracycline using molecularly imprinted polymer modified carbon nanotube-gold nanoparticles electrode. Electroanal. 2011;23:1863-1869. 
[20] Li JP, Li YP, Zhang Y, Wei G. Highly sensitive molecularly imprinted electrochemical sensor based on the double amplification by an inorganic Prussian blue catalytic polymer and the enzymatic effect of glucose oxidase. Anal Chem. 2012;84:1888-1893.

[21] Jing T, Niu JW, Xia H, Dai Q, Zheng HY, Hao QL, Mei SR, Zhou YK. Online coupling of molecularly imprinted solid-phase extraction to HPLC for determination of trace tetracycline antibiotic residues in egg samples. J Separat Sci. 2011;34:1469-1476.

[22] Hu XG, Pan JL, Hu YL, Huo Y, Li GK. Preparation and evaluation of solid-phase microextraction fiber based on molecularly imprinted polymers for trace analysis of tetracyclines in complicated samples. J Chromatogr A 2008;1188:97-107.

[23] Lv YK, Wang LM, Yang L, Zhao CX, Sun HW. Synthesis and application of molecularly imprinted poly(methacryclic acid)-silica hybrid composite material for selective solid-phase extraction and high-performance liquid chromatography determination of oxytetracycline residues in milk. J Chromatogr A. 2012;1227:48-53.

[24] Moreira FTC, Kamel AH, Guerreiro JRL, Sales MGF. Man-tailored biomimetic sensor of molecularly imprinted materials for the potentiometric measurement of oxytetracycline. Biosens Bioelectron. 2010;26:566-574.

[25] Qu G, Zheng S, Liu Y, Xie W, Wu A, Zhang D. Metal ion mediated synthesis of molecularly imprinted polymers targeting tetracyclines in aqueous samples. J Chromatogr B. 2009;877:3187-3193.

[26] Guerreiro JRL, Freitas V, Sales MGF. New sensing materials of molecularlyimprinted polymers for the selective recognition of chlortetracycline. Microchem J. 2011;97:173-181. 
[27] Zhao CY, Dai JD, Zhou ZP, Dai XH, Zou YL, Yu P, Zon TB, Li CX, Yan YS. One-pot method for obtaining hydrophilic tetracycline-imprinted particles via precipitation polymerization in ethanol. J Appl Polym Sci. 2014;131:40071.

[28] Lu ZY, Huo PW, Luo YY, Liu XL, Wu D, Gao X, Li CX, Yan YS. Performance of molecularly imprinted photocatalysts base on fly-ash cenospheres for selective photodegradation of single and ternary antibiotics solution. J Mol Catal A-Chem. 2013;378:91-98.

[29] Chen LG, Liu J, Zeng QL, Wang H, Yu AM, Zhang HQ, Ding L. Preparation of magnetic molecularly imprinted polymer for the separation of tetracycline antibiotics from egg and tissue samples. J Chromatogr A. 2009;1216:3710-3719.

[30] Jing T, Wang Y, Dai Q, Xia HA, Niu JW, Hao QL, Mei SR, Zhou YK. Preparation of mixed-templates molecularly imprinted polymers and investigation of the recognition ability for tetracycline antibiotics. Biosens Bioelectron. 2010;25:2218-2224.

[31] Suedee R, Srichana T, Chuchome T, Kongmark U. Use of molecularly imprinted polymers from a mixture of tetracycline and its degradation products to produce affinity membranes for the removal of tetracycline from water. J Chromatogr B. 2004;811:191200.

[32] Wang LQ, Lin FY, Yu LP. A molecularly imprinted photonic polymer sensor with high selectivity for tetracyclines analysis in food. Analyst. 2012;137:3502-3509.

[33] Sánchez-Polo M, Velo-Gala I, López-Peñalver JJ, Rivera-Utrilla J. Molecular imprinted polymer to remove tetracycline from aqueous solutions. Micropor Mesopor Mat. 2015;203:32-40. 
[34] Mu-Rong C, Chiung-Wen H, Jian-Lian C. Comparative synthesis of tetracyclineimprinted polymeric silicate and acrylate on CdTe quantum dots as fluorescent sensors. Biosens Bioelectron. 2014;61:471-477.

[35] Liu M, Li Y, Han J, Dong X. Synthesis of tetracycline-imprinted polymer microspheres by reversible addition-fragmentation chain-transfer precipitation polymerization using polyethylene glycol as a coporogen. J Separat Sci. 2014;37:11181125.

[36] Breton F, Rouillon R, Piletska EV, Karim K, Guerreiro A, Chianella I, Piletsky SA. Virtual imprinting as a tool to design efficient MIPs for photosynthesis-inhibiting herbicides. Biosens Bioelectron. 2006;22:1948-1954.

[37] Sánchez-Barragán I, Karim K, Costa-Fernández JM, Piletsky SA, Sanz-Medel A. A molecularly imprinted polymer for carbaryl determination in water. Sensor Actuat BChem. 2006;123:798-804.

[38] Tsyrulneva I, Zaporozhets O, Piletska E, Piletsky S. Molecular modelling and synthesis of a polymer for the extraction of amiloride and triamterene from human urine. Anal Methods. 2014;6:3429-3435.

[39] Lorenzo RA, Carro AM, Alvarez-Lorenzo C, Concheiro A. To remove or not to remove? The challenge of extracting the template to make the cavities available in molecularly imprinted polymers (MIPs). Int J Molec Sci. 2011;12:4327-4347.

[40] Richter BE, Jones BA, Ezzell JL, Porter NL, Avdalovic N, Phol C. Accelerated solvent extraction: A technique for sample preparation. Anal Chem. 1996;68:10331039. 
[41] Benito-Peña E, Martin S, Orellana G, Moreno-Bondi MC. Water-compatible molecularly imprinted polymer for the selective recognition of fluoroquinolone antibiotics in biological samples. Anal Bioanal Chem. 2009;393:235-245.

[42] Mojica ERE, Autschbach J, Bright FV, Aga DS. Tetracycline speciation during molecular imprinting in xerogel results in class-selective binding. Analyst. 2011;136:749-755.

[43] Yañez F, Chianella I, Piletsky SA, Concheiro A, Alvarez-Lorenzo C. Computational modeling and molecular imprinting for the development of acrylic polymers with high affinity for bile salts. Anal Chim Acta. 2010;659:178-185.

[44] Sing KSW, Everett DH, Haul RAW, Moscou L, Pierotti RA, Rouquérol J, Siemieniewska T. Reporting physisorption data for gas/solid systems with special reference to the determination of surface area and porosity. Pure Appl Chem. 1985;57:603-661.

[45] Umpleby RJ, Baxter SC, Rampey AM, Rushton GT, Chen Y, Shimizu KD. Characterization of the heterogeneous binding site affinity distributions in molecularly imprinted polymer. J Chromatogr B. 2004;804:141-149.

[46] Hassani M, Lazaro R, Perez C, Condon S, Pagan R. Thermostability of oxytetracycline, tetracycline, and doxycycline at ultrahigh temperatures. J Agric Food Chem. 2008;56:2676-2680.

[47] Meier F, Elbert SM, Mizaikoff B. A novel approach for the direct determination of residual template molecules in molecularly imprinted polymer matrices. Anal Methods. $2012 ; 4: 2755-2758$.

[48] Spivak DA. Optimization, evaluation, and characterization of molecularly imprinted polymers. Adv Drug Del Rev. 2005;57:1779-1794. 
[49] Dai J, Zhou Z, Zhao C, Wei X, Dai X, Gao L, Cao Z, Yan Y. Versatile method to obtain homogeneous imprinted polymer thin film at surface of superparamagnetic nanoparticles for tetracycline binding. Ind Eng Chem Res. 2014;53:7157-7166.

[50] Alegakis AK, Tzatzarakis MN, Tsatsakis AM, Vlachonikolis IG, Liakou V. In vitro study of oxytetracycline adsorption on activated charcoal. J Environ Sci Health B. 2000;35:559-569.

[51] Cheng DH, Yang SK, Zhao Y, Chen J. Adsorption behaviors of oxytetracycline onto sediment in the Weihe River, Shaanxi, China. J Chem. 2013;2013:652930. 


\section{Figure captions}

Figure 1. Structure of oxytetracycline (OTC) and functional monomers of the virtual library used for molecular modeling.

Figure 2. OTC-AMPS complex (blue, N; white, C; red, O; cyan, H).

Figure 3. OTC and non-reacted monomers removed by means of PLE from the various MIPs and NIPs synthesized.

Figure 4. OTC adsorption isotherms in purified water, $0.9 \% \mathrm{NaCl}$ and $3.5 \% \mathrm{NaCl}$. Row (a) refers to "series a" polymers, and row (b) to "series b" polymers.

Figure 5. OTC retained after passing through $0.100 \mathrm{~g}$ of polymer, aliquots $(2 \mathrm{~mL})$ of OTC solution $0.0171 \mathrm{mg} \mathrm{mL}^{-1}$.

Figure 6. Accumulated amount of OTC retained by polymers inside SPE cartridges and OTC concentration remaining in the percolated water after passing successive $2 \mathrm{~mL}$ aliquots of OTC solution $0.0171 \mathrm{mg} \mathrm{mL}^{-1}$ (concentration indicated with the dashed line).

Figure 7. Oxytetracycline eluted (solid black line) from the MIPs and NIPs particles in the SPE cartridge and eluted percentage calculated on the total amount of OTC retained (dashed color line). The red line indicates the change in the elution solvent from methanol to a mixture of methanol:HCl 0.1M 50:50 v:v. Polymers prepared using (a) HEMA, (b) AMPS, and (c) AMPS+HEMA as functional monomers. 
Table 1. Estimated interaction energies $\left(\mathrm{Kcal} \mathrm{mol}^{-1}\right)$ between OTC and ten best monomers from the virtual library of monomers, screened by LEAPFROG ${ }^{\mathrm{TM}}$.

\begin{tabular}{lc}
\hline Monomer & Interaction Energy $\left(\right.$ Kcal mol $\left.^{-1}\right)$ \\
\hline N,N-Methylenebis(acrylamide) & -37.41 \\
AMPS & -34.49 \\
Ethyleneglycol methacrylate phosphate & -31.40 \\
Methacrylamide & -30.22 \\
N-(2-(phenylamino)ethyl) methacrylamide & -29.34 \\
HEMA & -28.49 \\
Acrylamide & -27.75 \\
N,N-diethylaminoethyl methacrylate & -25.80 \\
Allylamine & -25.72 \\
Itaconic acid & -25.58 \\
\hline
\end{tabular}


Table 2. Amounts of monomers and template used for the synthesis of MIPs and NIPs, given as both mass and mol\% in the mixture. In all cases, $0.030 \mathrm{~g}$ of AIBN and $3 \mathrm{~mL}$ of DMSO were added.

\begin{tabular}{lcccc}
\hline Polymer & $\begin{array}{c}\text { EGDMA } \\
\text { grams / mol \% }\end{array}$ & $\begin{array}{c}\text { HEMA } \\
\text { grams / mol \% }\end{array}$ & $\begin{array}{c}\text { AMPS } \\
\text { grams / mol \% }\end{array}$ & $\begin{array}{c}\text { OTC } \\
\text { grams / mol \% }\end{array}$ \\
\hline 1a-NIP & $2.355 / 89.06$ & $0.190 / 10.94$ & $0 / 0$ & $0 / 0$ \\
1b-NIP & $2.355 / 89.06$ & $0.190 / 10.94$ & $0 / 0$ & $0 / 0$ \\
2a-MIP & $2.355 / 88.06$ & $0.190 / 10.82$ & $0 / 0$ & $0.075 / 1.12$ \\
2b-MIP & $2.355 / 88.06$ & $0.190 / 10.82$ & $0 / 0$ & $0.075 / 1.12$ \\
3a-NIP & $2.730 / 95.29$ & $0 / 0$ & $0.141 / 4.71$ & $0 / 0$ \\
3b-NIP & $2.730 / 91.01$ & $0 / 0$ & $0.282 / 8.99$ & $0 / 0$ \\
4a-MIP & $2.730 / 94.31$ & $0 / 0$ & $0.141 / 4.66$ & $0.075 / 1.03$ \\
4b-MIP & $2.730 / 90.11$ & $0 / 0$ & $0.282 / 8.90$ & $0.075 / 0.99$ \\
5a-NIP & $2.355 / 84.73$ & $0.190 / 10.41$ & $0.141 / 4.85$ & $0 / 0$ \\
5b-NIP & $2.355 / 80.81$ & $0.190 / 9.93$ & $0.282 / 9.26$ & $0 / 0$ \\
6a-MIP & $2.355 / 83.83$ & $0.190 / 10.30$ & $0.141 / 4.80$ & $0.075 / 1.06$ \\
6b-MIP & $2.355 / 79.99$ & $0.190 / 9.83$ & $0.282 / 9.16$ & $0.075 / 1.02$ \\
\hline
\end{tabular}


Table 3. Amount of OTC removed ( $\mathrm{mg} \mathrm{g}^{-1}$ polymer) through the different extractions carried out (1-with methanol in PLE; 2- with methanol/HCl in Falcon tubes) and total OTC removed (NIPs signal is due to non-reacted monomers); and results of nitrogen adsorption porosimetry analysis of the polymers.

\begin{tabular}{ccccccc}
\hline Polymer & $\begin{array}{c}\text { OTC } \\
\text { removed 1 } \\
\left(\mathbf{m g ~ g}^{-1}\right)\end{array}$ & $\begin{array}{c}\text { OTC } \\
\text { removed 2 } \\
\left(\mathbf{m g ~ g}^{-1}\right)\end{array}$ & $\begin{array}{c}\text { OTC total } \\
\text { removed }\end{array}$ & $\begin{array}{c}\text { Specific } \\
\text { surface area } \\
\left(\mathbf{m}^{2} \mathbf{g}^{-1}\right)^{\mathbf{b}}\end{array}$ & $\begin{array}{c}\text { Pore volume } \\
\left(\mathbf{c m}^{\mathbf{3}} \mathbf{g}^{-1}\right)\end{array}$ & $\begin{array}{c}\text { Average } \\
\text { pore } \\
\text { diameter } \\
(\mathbf{n m})\end{array}$ \\
\hline 1a-NIP & 0.02 & $0.09^{\mathrm{a}}$ & 0.11 & $375.6(4.4)$ & 0.53 & 7.36 \\
1b-NIP & 0 & $0.78^{\mathrm{a}}$ & 0.78 & $374.1(3.6)$ & 0.60 & 7.91 \\
2a-MIP & 18.20 & 0 & 18.20 & $322.3(3.6)$ & 0.43 & 6.70 \\
2b-MIP & 17.60 & 0.13 & 17.73 & $356.0(2.3)$ & 0.59 & 8.00 \\
3a-NIP & 0.06 & $0.36^{\mathrm{a}}$ & 0.42 & $437.4(4.6)$ & 0.59 & 7.66 \\
3b-NIP & 0.10 & $0.05^{\mathrm{a}}$ & 0.15 & $330.2(3.0)$ & 0.49 & 7.80 \\
4a-MIP & 8.50 & 2.44 & 10.94 & $392.5(4.1)$ & 0.65 & 8.86 \\
4b-MIP & 4.94 & 1.33 & 6.27 & $258.7(1.8)$ & 0.42 & 7.82 \\
5a-NIP & 0.05 & $0.11^{\mathrm{a}}$ & 0.16 & $354.8(3.6)$ & 0.60 & 8.47 \\
5b-NIP & 0.01 & $0.06^{\mathrm{a}}$ & 0.07 & $273.7(2.6)$ & 0.44 & 8.01 \\
6a-MIP & 7.51 & 3.20 & 10.71 & $280.4(3.2)$ & 0.43 & 7.63 \\
6b-MIP & 5.67 & 2.88 & 8.55 & $194.0(0.9)$ & 0.41 & 8.66 \\
\hline
\end{tabular}

${ }^{\mathrm{a}} \mathrm{NIPs}$ signal is due to non-reacted monomers. ${ }^{\mathrm{b}}$ Mean values and in parenthesis standard deviations $(\mathrm{n}=3)$. 
Table 4. Results from the fit of isotherms showed in Figure 4 to the Freundlich model.

\begin{tabular}{cccccccccc}
\hline \multirow{2}{*}{ Polymer } & \multicolumn{3}{c}{ Purified water } & \multicolumn{3}{c}{ 0.9\% NaCl } & \multicolumn{3}{c}{ 3.5\% NaCl } \\
& $m$ & $\log a$ & $R$ & $m$ & $\log a$ & $R$ & $m$ & $\log a$ & $R$ \\
\hline 1a-NIP & 0.942 & -0.902 & 0.940 & 1.139 & -1.118 & 0.948 & 0.778 & -0.354 & 0.827 \\
1b-NIP & 0.911 & -0.771 & 0.905 & 1.054 & -0.288 & 0.971 & 0.688 & -0.055 & 0.984 \\
2a-MIP & 0.854 & -0.708 & 0.971 & 1.009 & -0.942 & 0.962 & 0.682 & -0.092 & 0.944 \\
2b-MIP & 0.824 & -0.641 & 0.943 & 1.036 & -0.155 & 0.955 & 0.789 & -0.229 & 0.942 \\
3a-NIP & 0.365 & 1.512 & 0.879 & 0.504 & 0.604 & 0.914 & 0.550 & 0.520 & 0.922 \\
3b-NIP & 0.192 & 1.816 & 0.953 & 0.767 & 0.114 & 0.964 & 0.907 & -0.228 & 0.954 \\
4a-MIP & 0.367 & 1.426 & 0.868 & 0.613 & 0.185 & 0.927 & 0.728 & 0.054 & 0.930 \\
4b-MIP & 0.322 & 1.609 & 0.954 & 0.739 & 0.036 & 0.946 & 0.916 & -0.388 & 0.985 \\
5a-NIP & 0.356 & 1.557 & 0.840 & 0.577 & 0.431 & 0.888 & 0.399 & 0.675 & 0.774 \\
5b-NIP & 0.350 & 1.648 & 0.946 & 0.776 & 0.142 & 0.953 & 1.027 & -0.489 & 0.996 \\
6a-MIP & 0.385 & 1.424 & 0.946 & 0.755 & -0.192 & 0.933 & 0.863 & -0.554 & 0.866 \\
6b-MIP & 0.394 & 1.534 & 0.911 & 1.183 & -0.700 & 0.972 & 1.359 & -1.141 & 0.974 \\
\hline
\end{tabular}


Figure 1<smiles>CN(C)[C@H]1C(O)=C(C(N)=O)C(=O)[C@]2(O)C(=O)C3=C(O)c4c(O)cccc4[C@@](C)(O)[C@@H]3[C@H](O)[C@]12O</smiles>

Oxytetracycline (OTC)<smiles>C=CC(=O)O</smiles>
Acrylic acid<smiles>C=C(C)C(=O)O</smiles><smiles>C=Cn1cncc1I</smiles>

2-Hydroxyethyl methacrylate<smiles>C=Cc1ccncc1</smiles>

4-Vinylpyridine 2-Vinylpyridine

2-Acrylamide-2-methyl-<smiles>C=CC(=O)NC(C(C)C)S(=O)(=O)O[14C](C)(C)C(C)(C)C</smiles>

1-propanesulfonic acid (AMPS) 2-(Trifluoromethyl)acrylic

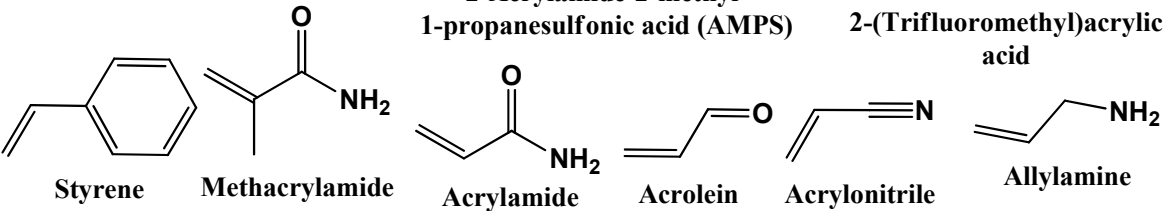<smiles>C=Cc1ccc(C=C)cc1</smiles>

p-Divinylbenzene Ethyleneglycol dimethacrylate<smiles>C=Cc1cccc(C=C)c1</smiles><smiles>C=CC(=O)NCNC(=O)C=C</smiles>

$\mathrm{N}, \mathrm{N}-$ Methylenebis(acrylamide)<smiles>O=C(O)/C=C/c1c[nH]cn1</smiles><smiles>C=C(C)C(=O)OCCN(CC)CC</smiles><smiles>C=C(C)C(=O)OCC</smiles><smiles>C1CCOC1</smiles>

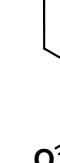<smiles>C=C(C)C=O</smiles>

Urocanic acid

N,N-Diethylaminoethyl methacrylate

Trimethylolpropane trimethacrylate<smiles>C=C(C)C(=O)NCCNc1ccccc1</smiles><smiles>C=C(C)C(=O)OCCOP(=O)(O)O</smiles>

N-(2-(Phenylamino)ethyl)methacrylamide

Ethyleneglycol methacrylate phosphate 
Figure 2

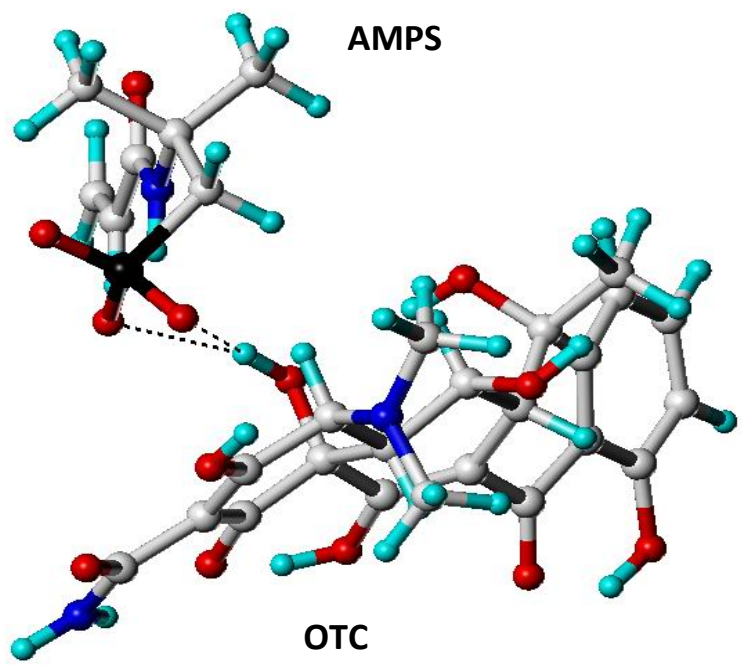


Figure 3
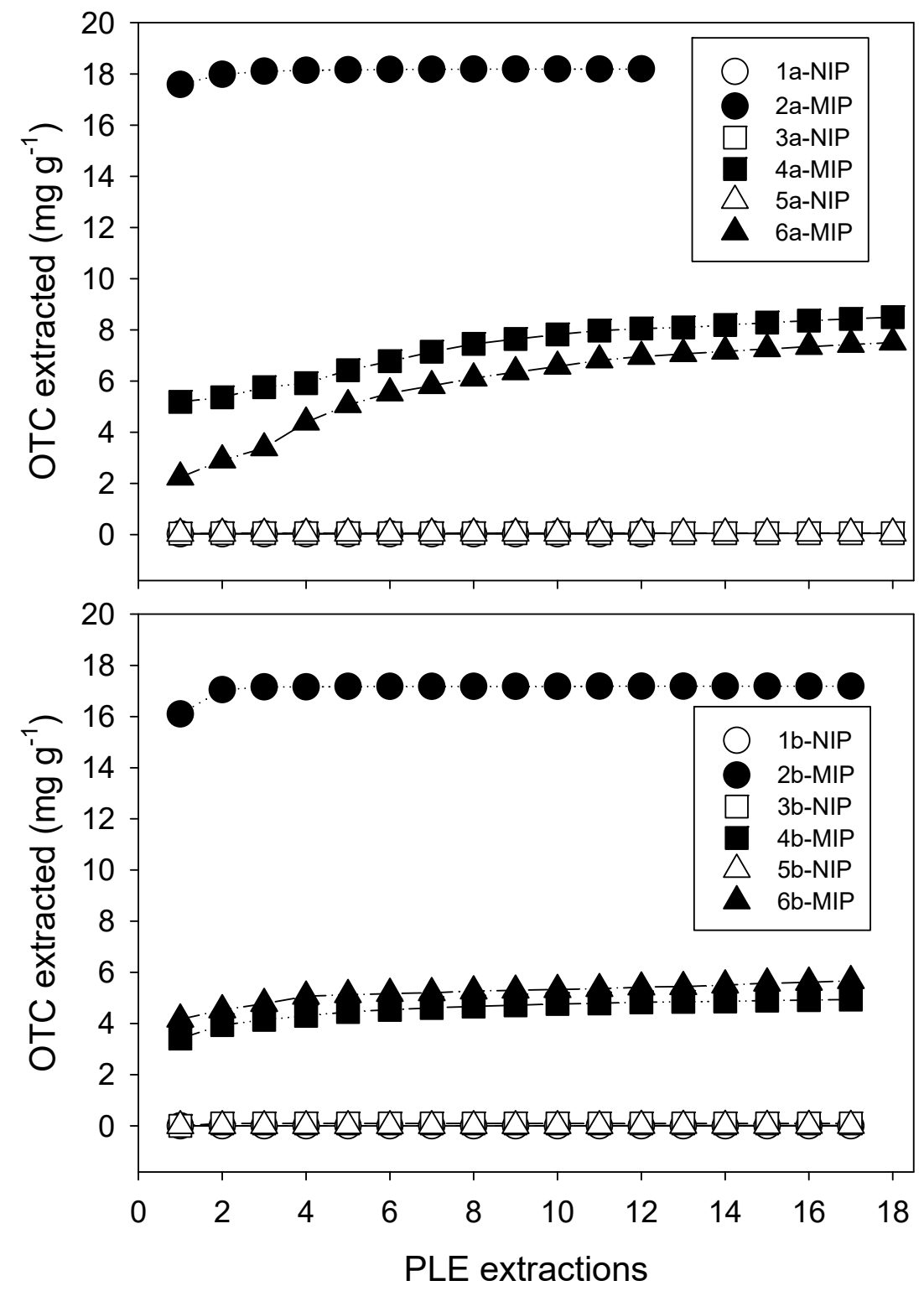
Figure 4
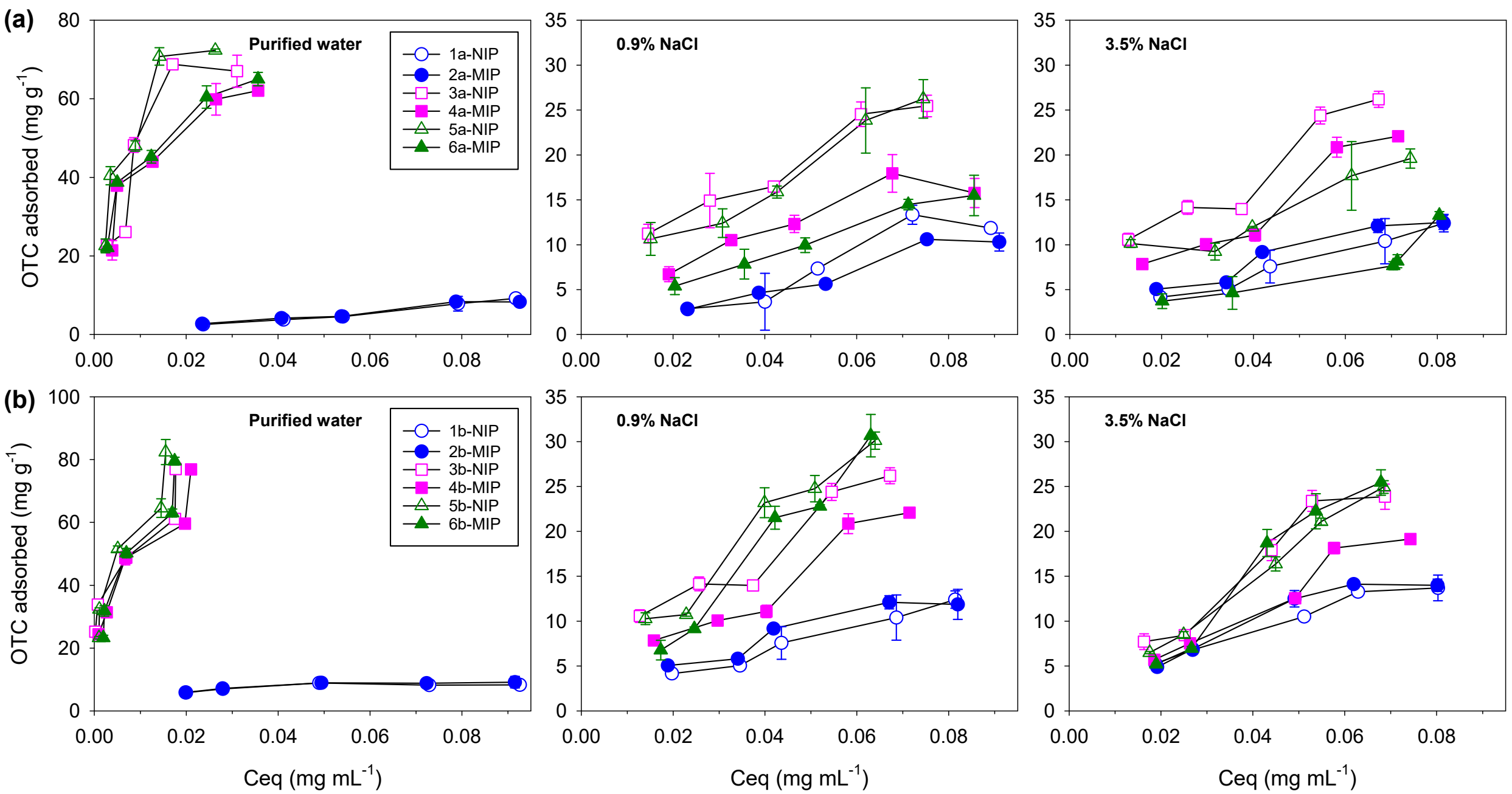
Figure 5

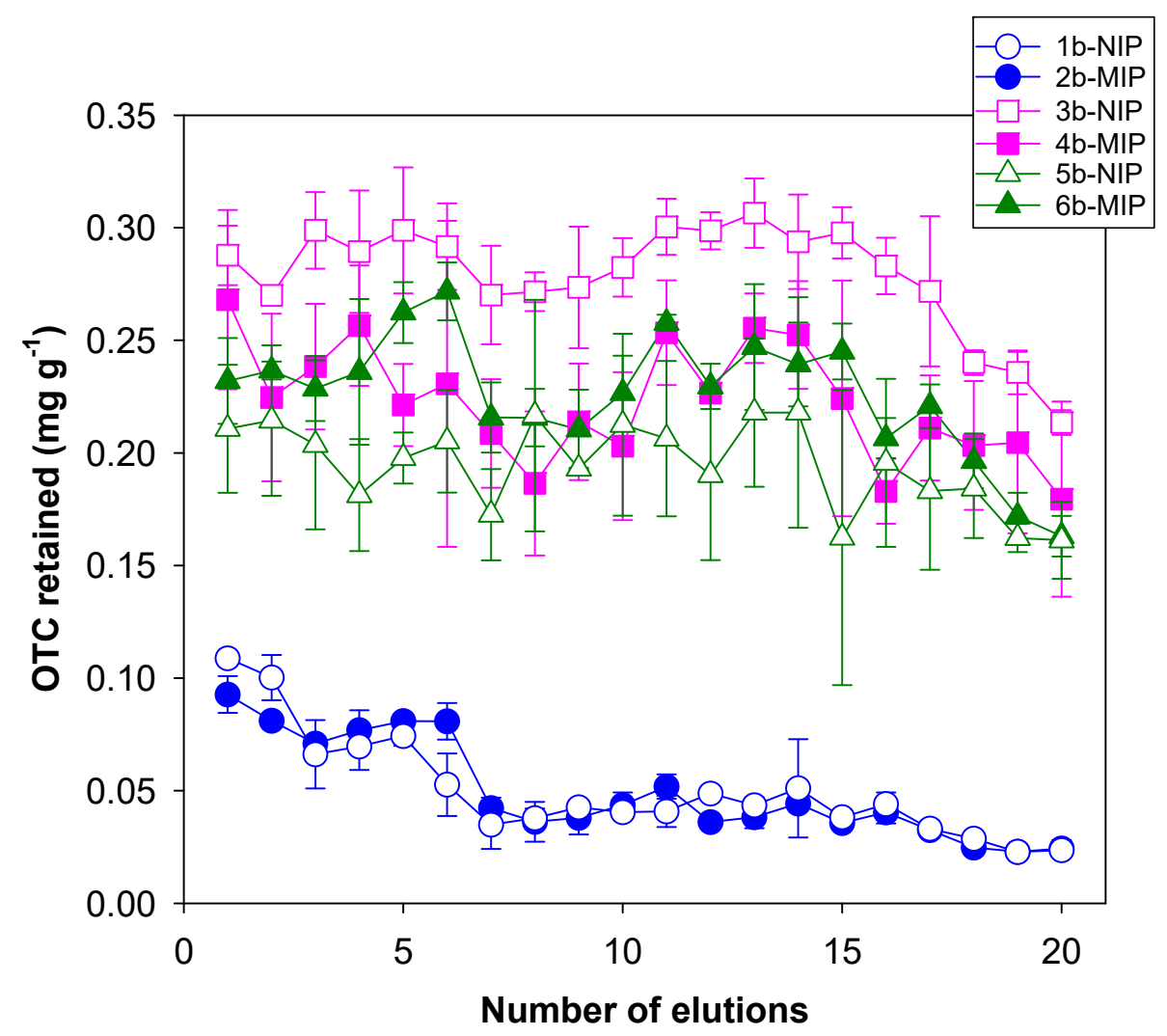


Figure 6
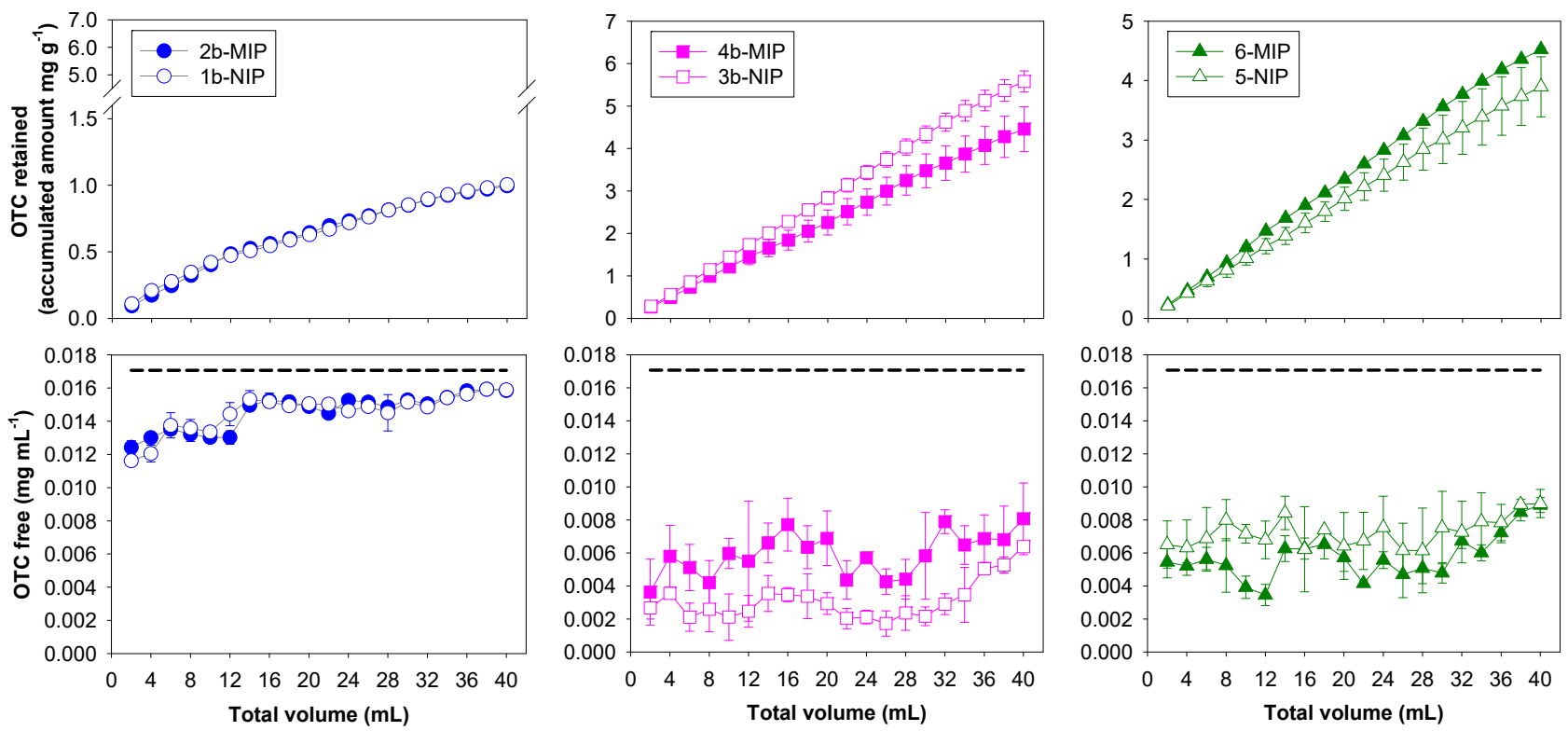
Figure 7
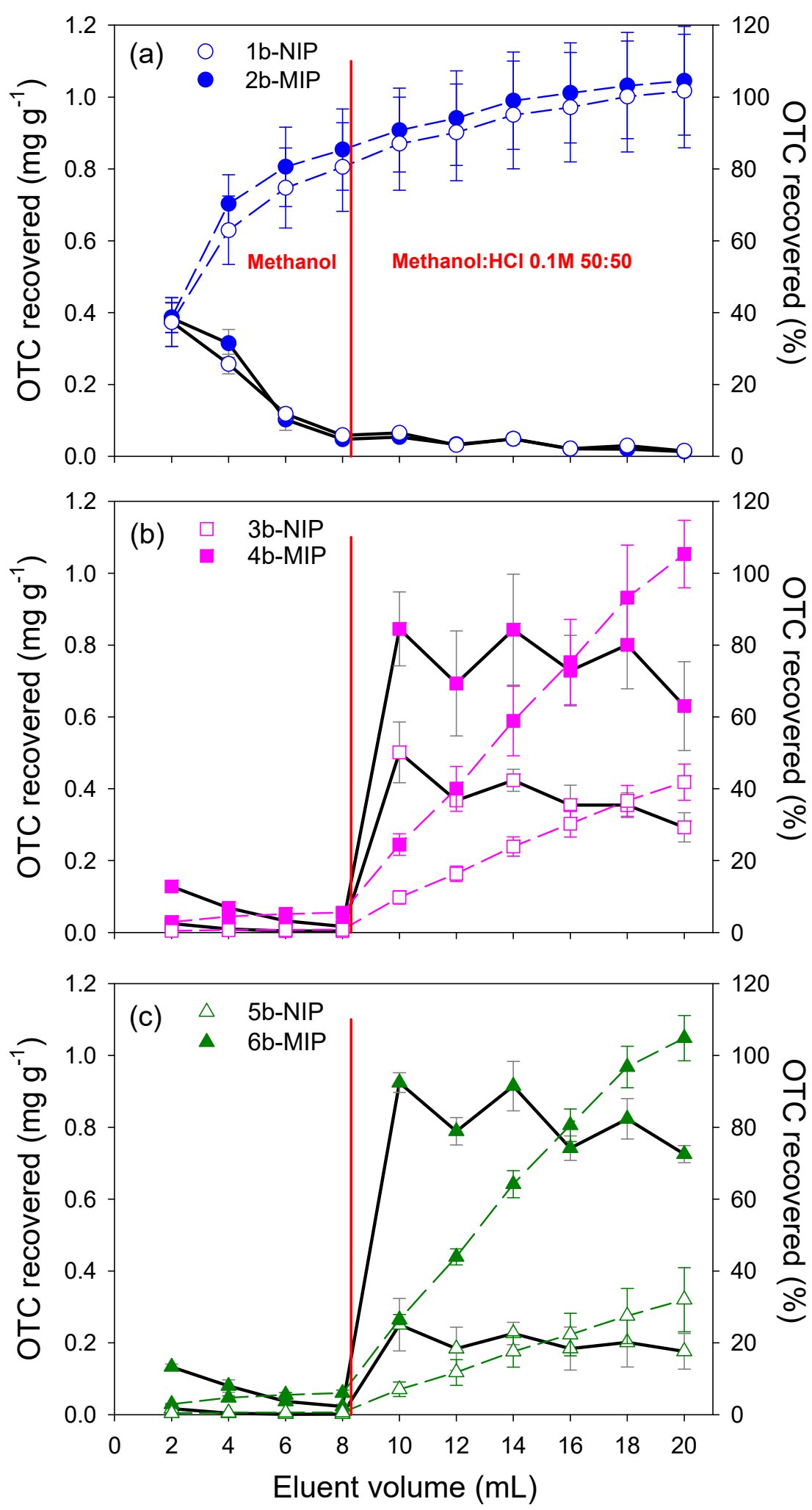
2016-08-03

\section{Oxytetracycline recovery from aqueous media using computationally designed molecularly imprinted polymers}

\section{Rodríguez Dorado, R.}

Springer Verlag (Germany)

Rodríguez-Dorado R, Carro AM, Chianella I, et al., Oxytetracycline recovery from aqueous media using computationally designed molecularly imprinted polymers, Analytical and Bioanalytical Chemistry, Vol. 408, Issue 24, September 2016, pp. 6845-6856 http://dx.doi.org/10.1007/s00216-016-9811-6 Downloaded from Cranfield Library Services E-Repository 